\section{Micro defense}

In plants and insects, small interfering RNA (siRNA) processed from viral double-stranded RNA functions in antiviral defense. In Science, Lecellier et al. now show that cellular 'microRNA' (miRNA) can mediate antiviral defense in mammalian cells. The miRNA miR-32 abrogates accumulation of the retrovirus primate foamy virus type 1 (PFV-1) in a human cell line. PFV-1, however, counteracts the effects of miR-32 using a silencing suppressor protein called Tas, which is also shown to function as a suppressor in other mammalian miRNA and plant siRNA pathways. These data demonstrate that cellular miRNA is involved in mammalian antiviral defense and may explain differences in virus permissivity, as the miRNA repertoire varies among cell types.

JDKW Science 308, 557-560 (2005)

\section{Targeting ATM}

Because human immunodeficiency virus 1 (HIV-1) induces DNA 'damage' in host cells during its life cycle, it may induce DNA repair responses that involve the ATM and ATR kinases. However, the relative importance of ATM in HIV-1 infection is contentious. In Nature Cell Biology, Lau et al. find that activation of the ATM-dependent DNA damage-response pathway is important in HIV-1 infection. Specific inhibition of ATM greatly reduces HIV-1 replication in cells by preventing efficient repair of damaged DNA, which leads to cell death. Targeting of host cell machinery that is not essential for cell survival, such as ATM, is advantageous because it prevents the replication of drug-resistant HIV-1 strains as efficiently as that of wild-type virus.

Nat. Cell Biol. (17 April 2005) doi:10.1038/ncb1250

\section{Healing cells}

Wound repair is associated with skin $\gamma \delta$ T cell-dependent keratinocyte proliferation, hyaluronan production in the extracellular matrix and migration of inflammatory cells into the damaged tissue. In the Journal of Experimental Medicine, Havren and colleagues investigate whether skin $\gamma \delta$ T cells mediate hyaluronan deposition and infiltration of inflammatory cells during this response. Mice deficient in $\gamma \delta$ T cells show defects in homing of macrophages to damaged tissue and delays in hyaluron deposition. This is due to reduced expression of hyaluron synthase by the epidermis. Specifically, $\gamma \delta$ T cells normally induce keratinocytes to synthesize hyaluronan via production of the keratinocyte growth factor FGF-7. Thus, application of hyaluronan or FGF-7 to wounds of $\gamma \delta$ T cell-deficient mice restores macrophage recruitment and shows that $\gamma \delta$ T cell-induced hyaluronan production by epithelial cells regulates inflammation.

$J D K W$

J. Exp.Med. 201, 1269-1279 (2005)

\section{Silencing nonsense}

Most mutant transcripts that contain premature stop codons are degraded by post-transcriptional processes known as nonsense-mediated mRNA decay. A report by Bühler et al. in Molecular Cell suggests that rearranged immunoglobulin genes that contain premature nonsense codons are

Written by Laurie A. Dempsey, Peter T. Lee and Jamie D.K. Wilson. recognized and silenced at the chromatin level. 'Nuclear run-on' experiments show that transcription rates are reduced for mutant immunoglobulin genes. This effect is correlated with histone modifications associated with inactive chromatin. Overexpression of the enzyme 3'hExo, which suppresses siRNA, could abrogate the silencing of mutant immunoglobulin minigenes. This work suggests recognition of immunoglobulin transcripts containing premature stop codons promotes active chromatin silencing, mediated in part by siRNA and other unknown factors. $L A D$ Mol. Cell 18, 307-317 (2005)

\section{Dousing inflammation}

IKK $\beta$, which forms part of the IKK enzyme complex that activates the transcription factor $\mathrm{NF}-\kappa \mathrm{B}$, is important in promoting inflammation, but the function of IKK $\alpha$ in innate immunity is unclear. In Nature, Lawrence et al. report that IKK $\alpha$ is also a negative regulator of macrophage activation and inflammation. Mice with defective IKK $\alpha$ show enhanced inflammation in response to bacterial infection and are more susceptible to septic shock. However, macrophages from mutant IKK $\alpha$ mice have greater bactericidal activity and are more resistant to apoptosis because of their increased NF- $\kappa \mathrm{B}$-dependent gene induction. IKK $\alpha$ seems to function by phosphorylating the two NF- $\mathrm{BB}$ subunits RelA and c-Rel, thereby promoting their degradation. Thus, IKK $\alpha$ negatively regulates inflammation by facilitating the removal of NF- $\kappa B$ from proinflammatory gene promoters.

Nature 434, 1138-1143 (2005)

\section{Bona fide receptors}

The C-type lectin dectin-1 acts in synergy with Toll-like receptor 2 to induce tumor necrosis factor (TNF) and interleukin 12 (IL-12) production. In Immunity, Reis e Sousa and colleagues now show that this pattern-recognition receptor can also directly signal gene transcription events. Stimulation of dectin-1 with its ligand induces tyrosine phosphorylation and Syk kinase recruitment to the receptor, resulting in IL-10 and IL-2 production. Syk-deficient dendritic cells show a block in IL-2 and IL-10 synthesis after dectin-1 stimulation, whereas dectin-Toll-like receptor $2-$ mediated TNF and IL-12 production is unaffected. These data indicate that dectin- 1 and perhaps other C-type lectins function as autonomous pattern-recognition receptors by directly inducing cytokine responses involved in innate immunity.

$J D K W$

Immunity 22, 507-517 (2005)

\section{Is Notch enough?}

Notch signaling is key in determining lineage fate choices. In Genes \& Development, Rothenberg and colleagues show that the kinetics and extent of expression by Notch-mediated target genes can be uncoupled from lineage-specific transcription factor expression in developing thymocytes. Fetal liver precursors cultured on OP9-DL1 stromal cells (expressing the Notch ligand Delta-like 1) maintain considerable lineage plasticity for several days despite their early expression of Hes1 and Dtx1, both Notch targets. Substantial numbers of progeny derived from single cells grown on OP9 stromal cells maintain their ability to develop into other lineages when culture conditions are switched after several days. Cells that commit early to the $\mathrm{T}$ cell lineage do not express more of the Notch target genes than cells that commit later. These data suggest Notch signals are not sufficient to specify $\mathrm{T}$ lineage commitment and that other factors are required.

Genes Dev. 19, 965-978 (2005)
$L A D$ 